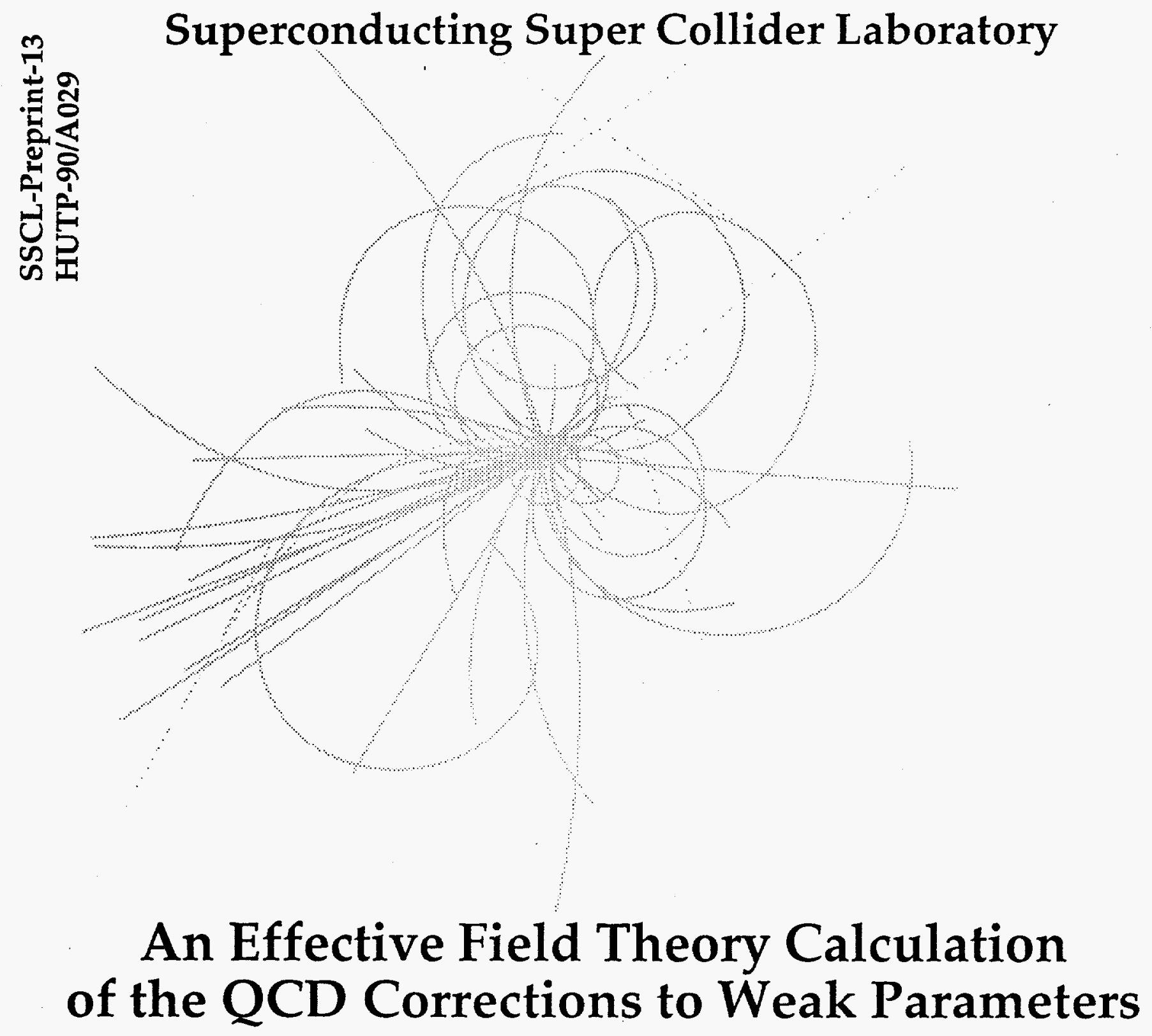

B. Grinstein and M. Wang

November 1991 
SSCL-Preprint-13

HUTP-90/A029

\title{
An Effective Field Theory Calculation of the QCD Corrections to Weak Parameters*
}

\author{
B. Grinstein ${ }^{a}$ \\ Superconducting Super Collider Laboratory ${ }^{b}$ \\ 2550 Beckleymeade Avenue \\ Dallas, TX 75237 \\ and \\ M. Wang ${ }^{c}$ \\ Lyman Laboratory of Physics \\ Harvard University \\ Cambridge, MA 02138
}

November 1991

\section{DISCLAIMER}

\begin{abstract}
This report was prepared as an account of work sponsored by an agency of the United States Government. Neither the United States Government nor any agency thereof, nor any of their employees, makes any warranty, express or implied, or assumes any legal liability or responsibility for the accuracy, completeness, or usefulness of any information, apparatus, product, or process disclosed, or represents that its use would not infringe privately owned rights. Reference herein to any specific commercial product, process, or service by trade name, trademark, manufacturer, or otherwise does not necessarily constitute or imply its endorsement, recommendation, or favoring by the United States Government or any agency thereof. The views and opinions of authors expressed herein do not necessarily state or reflect those of the United States Government or any agency thereof.
\end{abstract}

" Submitted to Nuclear Physics B.

a grinstein@sscvx1.bitnet or @sscvx1.ssc.gov

${ }^{b}$ Operated by the Universities Research Association, Inc., for the U.S. Department of Energy under Contract No. DE-AC35-89ER40486.

c mwang@huhepl.harvard.edu 


\section{DISCLAIMER}

Portions of this document may be illegible in electronic image products. Images are produced from the best available original document. 
HUTP-90/A029

SSCL-Preprint-13

\title{
An Effective Field Theory Calculation Of The QCD Corrections To Weak Parameters
}

\author{
Benjamin Grinstein ${ }^{\dagger}$
}

Superconducting Super Collider Laboratory, 2550 Beckleymeade Ave, Dallas TX 75237

and

Meng-Yuan Wang ${ }^{\ddagger}$

Lyman Laboratory of Physics, Harvard University, Cambridge, MA 02138

In the standard model the parameters $\rho$ and $M_{W}^{2} / M_{Z}^{2} \cos ^{2} \theta$ are unity at tree level. They are protected against large corrections by the so-called custodial symmetry. We investigate the symmetry breakin effect of effect of mass splittings in fermion doublets. The effective field theory method is applied to sum up the leading-log QCD corrections. We resolve a controversy concerning the scale at which the strong coupling constant is to be evaluated in the two-loop correction to electroweak parameters.

$11 / 91$

† grinstein@sscvx1.bitnet or @sscrx1.ssc.gov

† mwang@huhepl.harvard.edu 


\section{Introduction}

Precision measurements of weak interaction parameters give a detail test of our understanding of weak interactions physics. They are sensitive to extensions of and alternatives to the standard model, and are a source of information about the Higgs sector. It is therefore important to calculate all relevant radiative corrections to these parameters[1]. Several aspects of these corrections have been addressed in the past, both in[2]-[3] and beyond[4]-[5] the standard model.

In this paper we will address radiative corrections to $\rho$ and $M_{W}^{2} / M_{Z}^{2} \cos ^{2} \theta$, in the context of the standard model ${ }^{1}$. These parameters are protected from corrections by a "custodial" $S U(2)$-symmetry[7]. The symmetry is broken by several terms in the standard model lagrangian, and, in particular, by the mass difference between the components of a weak doublet. We will concentrate on this source of custodial-symmetry breaking. There are two cases of interest: either one quark mass is heavier than the $W$-boson and the other one is lighter, or both are heavier. We will consider both.

At one-loop, $\Delta \rho=\rho-1$ is given by $[8]$

$$
\Delta \rho=\frac{3 \alpha}{16 \pi M_{W}^{2} \sin ^{2} \theta_{W}}\left(m_{t}^{2}+m_{b}^{2}-\frac{2 m_{t}^{2} m_{b}^{2}}{m_{t}^{2}-m_{b}^{2}} \log \frac{m_{t}^{2}}{m_{b}^{2}}\right) .
$$

Since the mass splitting of the third generation of quarks, $m_{t}-m_{b}$, is large, $\Delta \rho$ is dominated by the first term (that is, the $m_{t}^{2}$ term). The large logarithm makes the last term an order of magnitude larger than the middle one. This suggests that, if experimental precision calls for the inclusion of the subleading terms in eq. (1.1), one should consider higher order diagrams which involve higher powers of the large log. Of course, there are correspondingly higher powers of the coupling constant. It is therefore pertinent to retain only the higher order corrections that involve powers of the strong coupling constant, $\alpha_{\mathbf{4}}$.

There is a standard method for resummation of a series of powers of $\alpha_{b} \log \left(m_{t} / m_{b}\right)$ (for an elementary introduction, see ref. [9]). One constructs an effective field theory by successively integrating out the heavy particles, i.e., those that contribute to low energy processes only as virtual particles. The effects of the heavy particles are contained in the coupling constants of an effective (non-renormalizable) hamiltonian for the light degrees of freedom. This method has been applied before to the calculation of $\Delta \rho[10]$, in the case

1 We will consider only the so-called "oblique" corrections, i.e., to the gauge boson propagators, not to the vertices, since other are process dependent[2][6]. 
where both the heavy quark mass, $m_{h}$, and the light one, $m_{l}$, are larger than the $W$ mass: $m_{h} \gg m_{l} \gg M_{W}$. In this work, we extend the result of ref. [10] to the case of interest, namely, $m_{h} \gg M_{W} \gg m_{l}$.

The effective theory technique allows for a systematic treatment of corrections. The importance of this becomes evident from the following consideration. There is some controversy in the literature as to how to incorporate into (1.1) corrections of order $\alpha_{s}$ (see, for example, the review in [11]). These may be numerically more important than the leading logs that we consider here, since they involve corrections to the first (order $m_{t}^{2}$ ) term. The problem is that it is not clear what the argument of the running coupling constant, $\bar{\alpha}_{s}$ should be: $m_{t}, M_{W}$, or $m_{b}$ ? Since $\bar{\alpha}_{\bullet}\left(m_{b}\right) / \vec{\alpha}_{s}\left(M_{W}\right) \sim 2$, this introduces an uncertainty in the correction of at least $100 \%$. The calculation in the effective field theory framework resolves the issue. The term in question is simply the first sub-leading log correction. In section 5 we elaborate on this point.

If there exists a fourth generation of quarks, then it is likely that both are more massive than the $W$. If their masses are vastly different, then $\Delta \rho$ already constrains the heavier to have a mass less than a few times $M_{W}$. Then, in fact, one doesn't have a very large hierarchy of masses, and we can only write $m_{h}>m_{l}>M_{W}$. One can compute $\Delta \rho$ again by constructing an effective field theory, which gives $\Delta \rho$ as an expansion in powers of light over heavy masses. This is similar to the case considered in ref. [10], but now with the additional complication that the first few powers of $m_{l}^{2} / m_{h}^{2}$ cannot be neglected. Before QCD corrections are incorporated, this is equivalent to expanding eq. (1.1) in powers of $m_{l}^{2} / m_{h}^{2}$. We address the question of how to incorporate QCD corrections to the term of order $m_{l}^{4} / m_{h}^{2}$, and we compute them. This, of course, is mostly of academic interest, since the log is not large. Still, there are several reasons why we think the exercise is interesting. As above, the effective field theory formalism is a starting point for a systematic expansion. Also, this exercise furnishes an example of the observation made in ref. [12], that corrections of order $m_{l}^{2} / m_{h}^{2}$ do not introduce new operators into the effective theory.

It is very likely that the precision in the measurement of the mass of the charged vector boson, $M_{W}$, will improve in the near future. It is therefore interesting to consider also corrections to the relation $M_{W}^{2} / M_{Z}^{2} \cos ^{2} \theta=1$. We have computed these in the same two cases as described above for $\Delta \rho$.

The paper is organized as follows. In section 2 we use the effective field theory to calculate $\Delta \rho$ with QCD turned off, and recover eq. (1.1), as expected. In section 3 we take into account the QCD effects, and sum up the leading logs, extending the result 
of ref. [10] to the physicaly interesting case $m_{b} \ll M_{W} \ll m_{t}$. In section 4 , the QCD corrected contribution of the $b$ - $t$ doublet to $M_{W \text {,phys }}^{2}$ is calculated. In section 5 , by the way of conclusions, we discuss the sub-leading corrections.

\section{2. $\Delta \rho$ without $\mathrm{QCD}$}

The $\rho$ parameter is defined as the ratio of the coefficient of the neutral current-current interaction to that of the charged current-current interaction in the four-fermion weak interaction lagrangian[10],

$$
\mathcal{L} \propto\left(j_{c}{ }^{\mu} j_{c \mu}^{\dagger}+\rho j_{0}{ }^{\mu} j_{0_{\mu}}\right) .
$$

Here $j_{c}$ stands for the charged current (that couples to the $W$-boson, with "charge" $\tau^{+}$) and $j_{0}$ the neutral current (that couples to the $Z$-boson, with "charge" $\tau^{3}-\sin ^{2} \theta_{W} Y$ ). With this definition $\rho$ can be measured directly from neutrino-hadron scattering.

In the standard model there is an accidental approximate global $S U(2)$-symmetry[7] under which $W^{a}$, for $a=1, \ldots, 3$, transform like a triplet. If this so-called "custodial" symmetry were exact then obviously $\rho=1$ identically. Therefore, only those interactions that break this custodial symmetry will contribute to $\Delta \rho$. Both hypercharge and the Higgs sector interactions break the symmetry, and these effects have been studied elsewhere[2]. Since we are interested in incorporating QCD effects, we now concentrate on corrections that arise from the mass splitting in the quark doublets. Moreover we will neglect the effects of flavor mixing, i.e., we will set all of the Cabibbo-Kobayashi-Maskawa angles to zero.

The four-fermion lagrangian in eq. (2.1) is the low energy effective lagrangian of the standard model. As described in the introduction, one can obtain this effective lagrangian starting from the standard model by integrating out one heavy particle after another. One thus arrives at an effective lagrangian with the four fermion interactions as the leading order terms in a series expansion with respect to the inverse of the masses of the heavy particles.

Consider now the contribution to $\Delta \rho$ from one heavy quark doublet $\left(\begin{array}{c}h \\ l\end{array}\right)$, with masses $m_{h} \gg m_{l} \gg M_{W}$. After integrating out the heavy quarks, the resulting effective theory is almost the standard model with one less quark doublet. The corrections are in the form of additional non-renormalizable terms suppressed by the heavy masses, and some corrections to the coefficients of the renormalizable terms. Among the latter one has, possibly, 
corrections $\Delta M_{W^{ \pm}}^{2}$ and $\Delta M_{W^{3}}^{2}$ to the mass parameters $M_{W^{ \pm}}^{2}$ and $M_{W^{3}}^{2}$, respectively. The oblique, i.e., propagator, correction to $\rho$ due to the mass splitting of this quark doublet is then simply

$$
\Delta \rho=\frac{\Delta M_{W^{ \pm}}^{2}-\Delta M_{W^{3}}^{2}}{M_{W}^{2}} .
$$

The situation is more involved, and more interesting, when $m_{h} \gg M_{W} \gg m_{l}$. One must integrate out the heavy $W$-bosons before one integrates out the lighter quark. At this stage the effective theory will already contain some of the current-current interactions in eq. (2.1). The coefficient of these will, in fact, give the order $-m_{h}^{2}$ contribution to $\Delta \rho$, c.f., eq. (1.1). But there are additional interactions that one should not neglect. They correspond to six-fermion operators, two of which are the lighter quark $l$. When $l$ is integrated out, these operators lead to current-current interactions. These arise from diagrams where the l-quark from the six-fermion operator is self-contracted, i.e., a oneloop diagram. This, in fact, is the origin, in the effective theory language, of the log term in eq. (1.1).

Let us now look at the calculations in some detail.

\section{1. $m_{h}>m_{l}>m_{W}$}

The weak- $S U(2)$ gauge sector of the lagrangian is[4]

$$
\begin{aligned}
\mathcal{L}= & -\left(1+\delta Z_{W^{+}}\right) \partial^{\mu} W^{+\nu} \partial_{\mu} W_{\nu}^{-}+\left(M_{W}^{2}+\delta M_{W^{+}}^{2}\right) W^{+\mu} W_{\mu}^{-} \\
& -\frac{1}{2}\left(1+\delta Z_{W^{3}}\right) \partial^{\mu} W^{3 \nu} \partial_{\mu} W_{\nu}^{3}+\frac{1}{2}\left(M_{W}^{2}+\delta M_{W^{3}}^{2}\right) W^{3 \mu} W_{\mu}^{3} \\
& -\frac{1}{2}\left(1+\delta Z_{B}\right) \partial^{\mu} B^{\nu} \partial_{\mu} B_{\nu}+\frac{1}{2}\left(M_{B}^{2}+\delta M_{B}^{2}\right) B^{\mu} B_{\mu} \\
& -\left(\delta Z_{W^{3} B}\right) \partial^{\mu} B^{\nu} \partial_{\mu} W_{\nu}^{3}+\left(M_{W^{3} B}^{2}+\delta M_{W^{3} B}^{2}\right) B^{\mu} W_{\mu}^{3} \\
& +\mathcal{L}_{i n t},
\end{aligned}
$$

where $\delta Z$ 's and $\delta M_{W}^{2}$ 's are counterterms. The fermion interactions in $\mathcal{L}_{\text {int }}$ follow from the covariant derivative $D_{\mu}=\partial_{\mu}+i g \tau^{a} W_{\mu}^{a}+i g^{\prime} Y B_{\mu}+\ldots$ and are shown in fig. 1 . Note that our definition of $\delta M_{W}^{2}$ does not correspond to the standard mass renormalization but rather to a combination of mass and wave-function renormalizations.

The first step in the construction of the effective lagrangian is to integrate out the $h$ quark. It is convenient to choose a renormalization scale $\mu=m_{h}$, to avoid the introduction 
of large logarithms of the ratio $\mu / m_{h}$. The gauge sector of the effective lagrangian is

$$
\begin{aligned}
\mathcal{L}^{\prime}= & -\left(1+\Delta Z_{W^{+}}^{\prime}+\delta Z_{W^{+}}^{\prime}\right) \partial^{\mu} W^{+\nu} \partial_{\mu} W_{\nu}^{-}+\left(M_{W}^{2}+\Delta M_{W^{+}}^{2}+\delta M_{W^{+}}^{\prime 2}\right) W^{+\mu} W_{\mu}^{-} \\
& -\frac{1}{2}\left(1+\Delta Z_{W^{3}}^{\prime}+\delta Z_{W^{3}}^{\prime}\right) \partial^{\mu} W^{3 \nu} \partial_{\mu} W_{\nu}^{3}+\frac{1}{2}\left(M_{W}^{2}+\Delta M_{W^{3}}^{2}+\delta M_{W^{3}}^{\prime 2}\right) W^{3 \mu} W_{\mu}^{3} \\
& -\frac{1}{2}\left(1+\Delta Z_{B}^{\prime}+\delta Z_{B}^{\prime}\right) \partial^{\mu} B^{\nu} \partial_{\mu} B_{\nu}+\frac{1}{2}\left(M_{B}^{2}+\Delta M_{B}^{2}+\delta M_{B}^{\prime 2}\right) B^{\mu} B_{\mu} \\
& -\left(\Delta Z_{W^{3} B}^{\prime}+\delta Z_{W^{3} B}^{\prime}\right) \partial^{\mu} B^{\nu} \partial_{\mu} W_{\nu}^{3}+\left(M_{W^{3} B}^{2}+\Delta M_{W^{3} B}^{2}+\delta M_{W^{3} B}^{\prime 2}\right) B^{\mu} W_{\mu}^{3} \\
& +\mathcal{L}^{\prime}{ }_{i n t},
\end{aligned}
$$

where $\Delta Z$ 's and $\Delta M_{W}^{2}$ 's are finite corrections of order $\alpha$, and the $\delta Z^{\prime}$ and $\delta M^{\prime}$ are counterterms. They are determined by matching the renormalized 1PI 2-point functions calculated in the effective theory with those of the full theory. Notice that the fields are chosen not to be "properly" normalized. Thus, the weak coupling constant $g$ is not rescaled. The interactions in $\mathcal{L}_{\text {int }}^{\prime}$ are shown in fig. 2. The first diagram is the same $l-l-W$ vertex as in fig. 1. The second corresponds to new interactions,

$$
\mathcal{L}_{\text {int }}^{\prime}=\sum_{n} c^{(n)} \mathcal{O}^{(n)}+\cdots
$$

Here

$$
\mathcal{O}^{(n)}=\bar{l}_{L} W^{-} i \not \partial\left(-\partial^{2}\right)^{n-1} W^{+} l_{L}
$$

The coefficients $c^{(n)}$ are again determined by the requirement that 1PI 4-point functions match properly. This gives, to leading order in $g^{2}$,

$$
\left.c^{(n)}\right|_{\mu=m_{h}}=\frac{g^{2}}{2\left(m_{h}^{2}\right)^{n}}
$$

Because of the large dimensionful denominator the effects of these operators are rather suppressed.

The operators in eq. (2.6) are not gauge invariant. For each $n$, there are many gauge invariant operators which would give the same 4-point functions as those of (2.6). Since electroweak symmetry has been spontaneously broken, we are not terribly concerned with preserving explicit gauge invariance. For now, the operators in (2.6) will suffice. When we come to consider QCD corrections, we will be forced to consider a version of (2.6) which is gauge invariant with respect to color gauge transformations. 
As we saw in eq. (2.2), we need only $\Delta M_{W}^{2}$ 's to calculate $\Delta \rho$. Therefore, we may set the external momentum to zero (the coefficients of the kinetic terms are irrelevant). Evaluating the diagrams in fig. 3 and fig. 4, we have

$$
\begin{aligned}
& \left.\Delta M_{W^{ \pm}}^{2}\right|_{\mu=m_{h}}=k\left(m_{h}^{2}+m_{l}^{2}\right) \\
& \left.\Delta M_{W^{3}}^{2}\right|_{\mu=m_{h}}=0
\end{aligned}
$$

where

$$
k=\frac{N_{c} g^{2}}{64 \pi^{2}},
$$

and $N_{c}=3$ is the number of colors. Notice that the result contains no logarithms at all. In the effective lagrangian language, logarithms of ratios of disparate scales, such as the one in eq. (1.1), result from scaling the effective lagrangian between the two scales.

It is important to note that the matching condition in eq. (2.8a) is a sub-leading$\log$ contribuition, in the sense that it will not be enhanced by a big logarithm, as the "leading" term will be. Nevertheless, the $m_{h}^{2}$ term is numerically more important, since it is enhanced by powers of the large scale (relative to the "leading" term). More properly, one should analyze the contributions to $\Delta \rho$ order by order in inverse powers of $m_{h}^{2}$. The leading term in this expansion is given in (2.8a). This term constitutes the full leading-log order $m_{h}^{2}$ contribution, since no terms of order $m_{h}^{2}$ can be generated in the effective theory. On the other hand, in the spirit of the leading-log expansion one should drop the term of order $m_{l}^{2}$ in eq. (2.8a). We will retain it here only because by neglecting the running of the electroweak coupling constants we will manage to recover the full result of (1.1) directly from the effective theory calculation.

Now we apply the renormalization group equation to scale the $\Delta M_{W}^{2}$ 's down. We consider the operators

$$
\begin{aligned}
& \mathcal{O}^{(+-)}=W^{+\mu} W_{\mu}^{-} \\
& \mathcal{O}^{(3,3)}=W^{3 \mu} W_{\mu}^{3},
\end{aligned}
$$

in addition to the operators in eq. (2.6). The mass operator of eq. (2.10a) mixes under renormalization into the operators in eq. (2.6). The coefficients must scale to make the effective lagrangian scale invariant. Therefore, their anomalous dimensions are the negative 
transpose of the matrix of anomalous dimensions of the operators. The relevant one-loop Feynman diagrams are shown in fig. 5 and fig. 6. We find,

$$
\begin{aligned}
\mu \frac{d}{d \mu} \Delta M_{W^{ \pm}}^{2} & =\frac{3}{8 \pi^{2}}\left(m_{l}^{4} c^{(1)}+m_{l}^{6} c^{(2)}+\cdots\right) \\
& =4 k\left(\frac{m_{l}^{4}}{m_{h}^{2}}+\frac{m_{l}^{6}}{m_{h}^{4}}+\cdots\right) \\
\mu \frac{d}{d \mu} \Delta M_{W^{3}}^{2} & =-4 k m_{l}^{2}
\end{aligned}
$$

In going from $(2.11 a)$ to $(2.11 b)$ we have used the fact that, to the order we are working, the coefficients $c^{(i)}$ are scale independent: $\mu d / d \mu c^{(i)}=0$. It is easy to incorporate the effect of the infinite set of operators in (2.6). The result is that the series in (2.11b) sums up to $4 k m_{l}^{4} /\left(m_{h}^{2}-m_{l}^{2}\right)$. The equations are trivially integrated, if we ignore the running of $g$ resulting from photon exchange:

$$
\begin{aligned}
& \Delta M_{W^{ \pm}}^{2}(\mu)=k\left(m_{h}^{2}+m_{l}^{2}-\frac{2 m_{l}^{4}}{m_{h}^{2}-m_{l}^{2}} \log \frac{m_{h}^{2}}{\mu^{2}}\right) \\
& \Delta M_{W^{3}}^{2}(\mu)=2 k m_{l}^{2} \log \frac{m_{h}^{2}}{\mu^{2}}
\end{aligned}
$$

In the final step in the construction of the effective lagrangian, we integrate out the $l$-quark. Thus, one obtains a new effective lagrangian of the same form as in (2.4), but without terms involving the light quark. Again, the $\Delta M_{W}^{2}$ 's are determined by matching the IPI functions in both effective theories. The matching is trivial, however, because all relevant diagrams are proportional to $\log \mu^{2} / m_{l}^{2}$, which vanishes at the boundary, $\mu=m_{l}$. So (2.12) hold for the coefficients in the final effective theory, with $\mu=m_{l}$.

We can now use eq. (2.2) to find the correction to the $\rho$-parameter. Of course, the result is the same as in eq. (1.1). The method is rather clumsy for something as simple as reproducing the one-loop computation that leads to eq. (1.1). But, as stated in the introduction, the power of the method lies in the ability to re-sum large logs when QCD corrections are included.

\section{2. $m_{h}>M_{W}>m_{l}$}

In this case, we begin again by integrating out the heavy quark. The effective lagrangian (2.4) is still appropriate, with the coefficients $\Delta M$ still given by eq. (2.12). But, in this case, we next integrate out the $W$-bosons, obtaining an effective theory with only 
the $l$-quark in it. Of course, it is understood that any other light degrees of freedom are also present in this final effective lagrangian.

We can no longer read off the correction $\Delta \rho$ from the mass terms in the lagrangian. Instead, we go back to the definition of $\rho$ in eq. (2.1). The effective lagrangian contains local four-fermion operators which will directly contribute in eq. (2.1). It also contains six-fermion operators, with two of the six fermions being $l$-quarks,

$$
\mathcal{Q}^{(n)}=j_{\mu}^{\dagger} j_{\nu}\left(\bar{l}_{L} \gamma^{\mu} i \not \partial\left(-\partial^{2}\right)^{n-1} \gamma^{\nu} l_{L}\right)
$$

They are nothing but the operators $\mathcal{O}^{(n)}$ of last sub-section, with the $W$ 's replaced by the currents $j_{\mu}$, times zero momentum $W$-propagators. These operators can contribute to $\rho$ in eq. (2.1) through one loop graphs, as depicted in fig. 7. Notice that the coefficients have a factor of $1 / M_{W}^{4}$. This might be a bit surprising, since the effective hamiltonian in (2.1) has as a coefficient $1 / M_{W}^{2}$, i.e., a factor of the Fermi constant, $G_{F}$. The additional factor of $1 / M_{W}^{2}$ is in the correction to $\rho$, as can be seen in eq. (1.1).

Since the operators $\mathcal{Q}^{(n)}$ are related to the operators $\mathcal{O}^{(n)}$ of last subsection by a trivial replacement of, to the order we are working, noninteracting fields, it follows that they satisfy the same renormalization group equations. Therefore, eqs. (2.11) still hold, and we again reproduce eq. (1.1) exactly.

\section{3. $\Delta \rho$ with QCD}

In the previous section, we showed that the effective field theory reproduced the complete order $-g^{2}$ results of the full theory. The reason why we need the effective theory is that, when QCD is involved and there is a large mass hierarchy in the lagrangian, $m \ll M$, one often encounters the combination $\left(\alpha_{s} / \pi\right) \log (m / M)$, which is not much smaller than 1. Therefore, conventional perturbation theory breaks down. Instead, one can re-sum all terms of the form $\alpha_{a}^{n} \log ^{n}(m / M)$. The corrections are then the so-called sub-leading logs, of the form $\alpha_{a}^{1+n} \log ^{n}(m / M)$. In the full theory, this can only be done by summing an infinite series of diagrams of higher and higher order in the loop expansion. In the effective theory, a one-loop calculation automatically sums up the leading logs.

In the case at hand, the zeroth-order result of last section already contains a large logarithm. Therefore, by "leading logarithms" we will mean, in this context, terms with one less power of $\alpha$, than of the log. 


\section{1. $m_{h}>m_{l}>M_{W}$}

The calculation in this limit was first done in ref. [10]. We will reproduce that result there and we will extend it to higher orders in $m_{l} / m_{h}$. To begin with, there is no need to recalculate the matching at $m_{h}$. This was done above in one-loop order, and since this is $\alpha_{s}$ independent, there is no change in this case.

As explained above, the leading term, of order $k m_{h}^{2} / M_{W}^{2}$, is generated by matching at the heavier scale, but receives no corrections from running down to $\mu=m_{l}$. We will concentrate on the subleding terms since these do recieve nontrivial corrections from QCD.

The QCD effects arise, in this order, from the scaling of the coefficients in the effective lagrangian. There are two sources of QCD corrections. Firstly, one expects, on general grounds, the renormalization group equations (2.11) to be modified. In particular, in eq. (2.11a), which arises from mixing of the higher dimension operators in (2.6) into the mass operators, the coefficients $c^{(i)}$ may have non-trivial dependence on the scale $\mu$. At one-loop this is computed for the one gluon exchange diagrams of fig. 8. Secondly, the parameters of the effective lagrangian are also scale dependent. To the order we are working, this means we will have to incorporate into the solution of $(2.11 a)$ the scale dependence of the light quark mass parameter $m_{l}$ :

$$
m_{l}^{2}(\mu)=m_{l}^{2}\left(\mu_{0}\right)\left[\frac{\bar{\alpha}_{s}(\mu)}{\bar{\alpha}_{s}\left(\mu_{0}\right)}\right]^{\frac{24}{33-2 n_{f}}} .
$$

Here, $n_{f}$ is the number of quark flavors in the effective theory. Note that the running of other coupling constants, e.g., $g$, is higher order (in $g^{2}$ ) and will be neglected in our approximation.

It is interesting to note that, again on general grounds, to this order, the renormalization group equation for $M_{W^{3}}^{2}, c . f$., eq. $(2.11 c)$, is not modified by QCD corrections. The reason is that the operators $\mathcal{O}^{(n)}$ do not mix into the $W^{3}$-mass operators in the effective lagrangian (2.4). The running arises only from the graph in fig. 8. Therefore, we know everything (eqs. (2.11c) and (3.1)) we need to determine $\Delta \rho$ to order $k m_{l}^{2} / M_{W}^{2}$. This, in fact, was the calculation presented in ref. [10].

In order to determine $\Delta \rho$ to order $k m_{l}^{4} / \mathrm{m}_{h}^{2} M_{W}^{2}$, one must determine the coeficient function $c^{(1)}$ of eq. (2.11a). Either by direct calculation or by an indirect argument one finds that

$$
\mu \frac{d}{d \mu} c^{(1)}=0 .
$$


We will give here the indirect argument. Only the first operator, $\mathcal{O}^{(1)}$, contributes to the running of the function $c^{(1)}$. For the purpose of computing the QCD mixing, the $W$-fields in $\mathcal{O}^{(1)}$ are inert, as they are colorless. Moreover, derivatives acting on the $W$-fields can be neglected, as we are interested, eventually, in zero momentum Green functions. Thus the operator $\mathcal{O}^{(1)}$ can be thought of as a dimension-4 fermionic operator with two free indices:

$$
\mathcal{O}^{(1)^{\mu \nu}}=\bar{\psi} \gamma^{\mu} i \not D \gamma^{\nu} \psi
$$

The symmetric and antisymmetric pieces don't mix with each other. Moreover, the latter cannot mix with the $W$-mass term in the effective lagrangian. Therefore it is safely neglected, and we can write

$$
\mathcal{O}^{(1)^{\mu \nu}}=\bar{\psi} i\left(\gamma^{\mu} D^{\nu}+\gamma^{\nu} D^{\mu}-g^{\mu \nu} \not D\right) \psi
$$

It is obvious now that this can be separated into spin-2 and spin-0 pieces. They don't mix with each other, and the former does not mix into the $W$-mass term in the lagrangian. We are left with the spin-0 piece. But this is non other than the kinetic energy lagrangian of the fermion field (times the metric tensor $g^{\mu \nu}$ ). It is well known[13] that, at one loop order, the self renormalization of the kinetic energy operator is cancelled out exactly by the wavefunction renormalization of the fermion fields. Hence, the diagrams of fig. 8 give a vanishing contribution to the anomalous dimension matrix. All that remains is the mixing of $\mathcal{O}^{(1)}$ into the mass term, but this is precisely the calculation of section 2 above. This completes the argument leading to eq. (3.2).

We have shown that, to order $k\left(m_{l}^{4} / m_{h}^{2}\right)$, and in the leading-log approximation,

$$
\begin{aligned}
& \mu \frac{d}{d \mu} \Delta M_{W^{ \pm}}^{2}=4 k\left(\frac{m_{l}^{4}}{m_{h}^{2}}+\cdots\right) \\
& \mu \frac{d}{d \mu} \Delta M_{W^{3}}^{2}=-4 k m_{l}^{2}
\end{aligned}
$$

As explained above, $m_{l}^{2}$ is now a function of $\mu$ because it is also renormalized by gluon exchange. It is straightforward to integrate these equations. We find

$$
\begin{aligned}
& \left.\Delta M_{W \pm}^{2}\right|_{\mu=m_{l}}=k\left[m_{h}^{2}+\frac{24 \pi m_{l}^{4}\left(m_{h}\right)}{\bar{\alpha}_{s}\left(m_{h}\right) m_{h}^{2}\left(2 n_{f}+15\right)}\left(1-\left(\frac{\bar{\alpha}_{s}\left(m_{l}\right)}{\bar{\alpha}_{s}\left(m_{h}\right)}\right)^{\frac{2 n_{f}+15}{33-2 n_{f}}}\right)\right] \\
& \Delta M_{\left.W^{3}\right|_{\mu=m_{1}} ^{2}}^{2}=-k \frac{24 \pi m_{l}^{2}\left(m_{h}\right)}{\bar{\alpha}_{s}\left(m_{h}\right)\left(2 n_{f}-9\right)}\left(1-\left(\frac{\bar{\alpha}_{s}\left(m_{l}\right)}{\bar{\alpha}_{s}\left(m_{h}\right)}\right)^{\frac{2 m_{f}-9}{33^{-2 n_{f}}}}\right)
\end{aligned}
$$


For consistency we have dropped a term of order $m_{l}^{2}$ in (3.6a) that arises from the boundary condition, eq. (2.8a). Finally, substituting these results into (2.2), we get

$$
\begin{aligned}
\Delta \rho=\frac{k}{M_{W}^{2}}\left\{m_{h}^{2}\right. & +\frac{24 \pi}{\bar{\alpha}_{s}\left(m_{h}\right)}\left[\frac{m_{l}^{2}\left(m_{h}\right)}{2 n_{f}-9}\left(1-\left(\frac{\bar{\alpha}_{s}\left(m_{l}\right)}{\bar{\alpha}_{s}\left(m_{h}\right)}\right)^{\frac{2 \pi_{l}-9}{33-2 n_{f}}}\right)\right. \\
& \left.\left.+\frac{m_{l}^{4}\left(m_{h}\right)}{m_{h}^{2}\left(2 n_{f}+15\right)}\left(1-\left(\frac{\bar{\alpha}_{s}\left(m_{l}\right)}{\bar{\alpha}_{s}\left(m_{h}\right)}\right)^{\frac{2 m_{f}+15}{33-2 m_{f}}}\right)+\mathcal{O}\left(\frac{m_{l}^{6}}{m_{h}^{4}}\right)\right]\right\}
\end{aligned}
$$

For compactness, the result has been written in terms of $m_{l}\left(m_{h}\right)$. With the help of eq. (3.1), it can be rewritten in terms of the more physical renormalization group invariant parameter $m_{l}^{*}=m_{l}\left(m_{l}^{*}\right)$.

\section{2. $m_{h}>M_{W}>m_{l}$}

This case is physically interesting because it corresponds to the third generation quarks. As opposed to what we presented in the last sub-section, in this case we will concentrate only on the contribution to $\Delta \rho$ of order $k\left(m_{l}^{2} / M_{W}^{2}\right)$. This is, of course, the most important contribution in leading logs. Moreover, it was not computed in ref. [10].

There is very little new in this case. As in section 2.2 above, once the $W$ 's are integrated out, we need to consider operators $\mathcal{Q}^{(n)}$. But if we concentrate, as we do, in the corrections to $\rho$ that are not suppressed by powers of $m_{h}$, then the effect of these six-fermion operators can be neglected. Below the $W$-scale we therefore have the usual current-current interaction lagrangian. Explicitly,

$$
\mathcal{L}_{\text {int }}=t^{ \pm}(\mu) h_{\mu}^{+} \ell_{\mu}^{-}+t^{3}(\mu) h_{\mu}^{0} \ell_{\mu}^{0}+u(\mu) j_{\mu}^{0} \ell_{\mu}^{0}+v_{1}(\mu) j_{\mu}^{0} h_{\mu}^{0}+v_{2}(\mu) j_{\mu}^{a} h_{\mu}^{a}+\cdots
$$

Here $\ell_{\mu}$ are the leptonic currents, $h_{\mu}$ are the hadronic currents, excluding the $l$-quark, and $j_{\mu}^{0}$ is the l-quark neutral current. In the last term, the one with the coefficient $v_{2}$, the flavor-neutral currents are color octets, e.g.,

$$
j_{\mu}^{a}=\bar{l}_{L} T^{a} \gamma_{\mu} l_{L}
$$

We have included this term as we expect it to mix, under renormalization, with the $v_{1}$ term. The ellipses in eq. (3.8) stand for terms that are present at low energies but are irrelevant to our discussion, e.g., purely leptonic four fermion operators. We can use the results 
of the previous section to extract, at $\mu=M_{W}$, the coefficient $t^{ \pm}$of the charged-charged current term:

$$
\begin{aligned}
\left.t^{ \pm}\right|_{\mu=M_{W}} & =-\frac{g^{2}}{M_{W}^{2}+\Delta M_{W^{ \pm}}^{2}} \\
& =-\frac{g^{2}}{M_{W}^{2}+k\left(m_{h}^{2}+m_{l}^{2}\left(m_{h}\right)\right)} .
\end{aligned}
$$

Also, the coefficients of the 3-3 current terms are

$$
\begin{aligned}
\left.t^{3}\right|_{\mu=M_{W}} & =\left.u\right|_{\mu=M_{W}}=\left.v_{1}\right|_{\mu=M_{W}}=-\frac{g^{2} / 4}{M_{W}^{2}+\Delta M_{W^{3}}^{2}} \\
& =-\frac{g^{2}}{4}\left[M_{W}^{2}+k \frac{24 \pi m_{l}^{2}\left(M_{W}\right)}{\bar{\alpha}_{s}\left(M_{W}\right)\left(2 n_{f}-9\right)}\left(1-\left(\frac{\bar{\alpha}_{s}\left(m_{h}\right)}{\bar{\alpha}_{s}\left(M_{W}\right)}\right)^{\frac{2 \pi_{f}-9}{33-2 n_{f}}}\right)\right]^{-1} .
\end{aligned}
$$

Of course, we also have

$$
\left.v_{2}\right|_{\mu=M_{W}}=0 .
$$

Next, our task is to find the renormalization group equation satisfied by the coefficients $t(\mu), u(\mu)$ and $v(\mu)$. Only the scaling of $v(\mu)$ will involve explicit $\alpha_{s}$ dependence, since these are the coefficients of the four quark operators. One gluon exchange graphs, as in fig. 9 , give the equation

$$
\mu \frac{d}{d \mu}\left(\begin{array}{l}
v_{1} \\
v_{2}
\end{array}\right)=\frac{\alpha_{a}}{6 \pi}\left(\begin{array}{cc}
0 & 8 \\
9 & -6
\end{array}\right)\left(\begin{array}{l}
v_{1} \\
v_{2}
\end{array}\right)
$$

Additional equations, giving the mixing of these coefficients into $t(\mu)$, are $\alpha_{\mathrm{s}}$ independent and are therefore exactly the same as those found in section 2.2 , where the problem with QCD turned off was considered. Explicitly, one has that the charged current does not run,

$$
\mu \frac{d}{d \mu} t^{ \pm}=0
$$

while $t^{3}$ is renormalized by the second diagram in fig. 7 , which gives

$$
\mu \frac{d}{d \mu} t^{3}=-\frac{m_{l}^{2}}{4 \pi^{2}} v_{1} u
$$

One also has,

$$
\mu \frac{d}{d \mu} u=0 .
$$

This is correct if we neglect contributions to $\Delta \rho$ of order $k\left(m_{l}^{4} / M_{W}^{4}\right)$. 
Solving eqs. (3.13)-(3.15), one obtains

$$
\begin{aligned}
\Delta \rho=\frac{k}{M_{W}^{2}} & \left\{m_{h}^{2}-\frac{8 \pi m_{l}^{2}\left(M_{W}\right)}{\bar{\alpha}_{s}\left(M_{W}\right)}\left[\frac{3}{2 n_{f}-9}\left(1-\left(\frac{\bar{\alpha}_{s}\left(m_{h}\right)}{\bar{\alpha}_{s}\left(M_{W}\right)}\right)^{\frac{2 n_{f}-9}{33-2 n_{f}}}\right)\right.\right. \\
& -\frac{2}{2 n_{f}-3}\left(1-\left(\frac{\bar{\alpha}_{s}\left(m_{l}\right)}{\bar{\alpha}_{s}\left(M_{W}\right)}\right)^{\frac{2 n_{f}-3}{33-2 n_{f}}}\right) \\
& \left.\left.-\frac{1}{2 n_{f}-21}\left(1-\left(\frac{\bar{\alpha}_{s}\left(m_{l}\right)}{\bar{\alpha}_{s}\left(M_{W}\right)}\right)^{\frac{2 m_{l}-21}{33-2 n_{f}}}\right)\right]+O\left(\frac{m_{l}^{4}}{m_{h}^{2}}\right)\right\}
\end{aligned}
$$

\section{The Mass of the $W$.}

The $\rho$ parameter is one of many observables of the standard model. There are three independent parameters in the gauge sector of the standard model. It is customary to choose them to be three well measured observables: the Fermi constant as measured in muon decay, $G_{\mu}$, the fine structure constant, $\alpha$, and the mass of the neutral vector boson, $M_{Z}$. Any observable can be expressed in terms of these three. Of course, additional parameters are introduced as the model is extended to include, for example, quarks and leptons.

The methods used in previous sections can also be applied to calculate radiative corrections to the other observables of the standard model. In this section we will demonstrate how to calculate the mass of the $W$-boson. Again we will work in the leading-log approximation. We will see that radiative corrections to wavefunctions must be included, but there is no QCD component of these corrections. The effect of wavefunction renormalization could have been obtained by computing in the full theory and neglecting QCD effects. The reader is welcomed to add to the result below the subleading-log terms that can, actually, be numerically important.

We will concentrate on the physical case $m_{t}>M_{W}>m_{b}$. Not only will we include the custodial symmetry breaking due to the mass splitting between $t$ and $b$ quarks, but we will also include the explicit breaking from hypercharge. This is necesary since $W_{\mu}^{3}-B_{\mu}$ mixing occurs at tree level.

The calculation is different from that of sections 2 and 3 in several ways. First, of course, we have to compute wavefunction renormalization. Any wavefunction renormalization of the gauge fields is irrelevant to the calculation of $\Delta \rho$. The corrections $\Delta Z$ of 
eq. (2.4) do not show up in the expression for $\Delta \rho$, eq. (2.2), since only the zero momentum part of the self energy diagram is relevant. In contrast, the pole in the propagator will necessarily involve the self energy diagram at momenta of the order of the tree level masses. Thus we are led to repeat the argument of earlier sections, but now including the running of $\Delta Z$. Second, the dependence of $\Delta Z$ on $m_{t}$ is only logarithmic, as expected for a dimension 4 operator. Custodial symmetry breaking by quark masses enters the result through mild (logarithmic) dependence. It is the mixing of $W^{3}$ and $B$, the hypercharge gauge boson, that gives the leading custodial symmetry breaking contribution. Therefore, the heavy quark contribution to $\Delta Z$ is enhanced over that of light quarks only by a 'large' logarithm. Numerically the contributions of light quarks cannot be neglected[14]. We do not include them here as we are primarily interested in the issue of re-summing the 'large' logarithms when QCD is included. Third, since the external momentum is large, $p^{2}=M_{W}^{2}$, we avoid large logarithms in the self energy diagram by choosing a renormalization point of order of the large scale, $\mu \sim M_{W}$. There is no running below $M_{W}$, and the $b$-quark mass is irrelevant.

Other than for these points, the calculation is entirely analogous to that presented in previous sections. We therefore only sketch the method. With the sign convention for the self-energy part $\Gamma^{(2)}$ that the full propagator is

$$
-i g^{\mu \nu} \frac{1}{(1+\Delta Z) q^{2}-\left(M^{2}+\Delta M^{2}\right)-\Gamma^{(2)}\left(q^{2}\right)},
$$

the physical mass is given in terms of the lagrangian mass, at one-loop order, by

$$
M_{\mathrm{phys}}^{2}=M^{2}+\delta M^{2}
$$

where

$$
\delta M^{2}=-\Delta Z M^{2}+\Delta M^{2}+\operatorname{Re} \Gamma^{(2)}\left(M^{2}\right) .
$$

We will compute all three quantities, $\Delta M^{2}, \Delta Z$ and $\Gamma^{(2)}$ at the renormalization point $\mu=$ $M$. Of course, the resulting physical mass is $\mu$-independent. But choosing $\mu=M$ ensures that no large logarithms are present in $\Gamma^{(2)}\left(M_{\text {phys }}^{2}\right)$, making the computation reliable.

The mass shifts $\Delta M^{2}$ were computed in section 3.2. One must remember, though, that in this case we should choose $\mu=M$, as indicated above. We can read off the mass-shifts from eqs. (3.7) by replacing $\bar{\alpha}_{s}\left(m_{l}\right) \rightarrow \bar{\alpha}_{s}\left(M_{W}\right)$. 
We turn to the computation of $\Delta Z$ and $\Gamma^{(2)}$. We retain only the leading contribution as far as inverse powers of $m_{t}$ are concerned. Recall that we work in the leading-log approximation. The matching conditions are trivial, since they arise at 1-loop order:

$$
\begin{aligned}
\Delta Z_{\left.W^{ \pm}\right|_{m_{\imath}}} & =0 \\
\left.\Delta Z_{W^{3}}\right|_{m_{\imath}} & =0 \\
\left.\Delta Z_{B}\right|_{m_{\imath}} & =0 \\
\left.\Delta Z_{W^{3} B}\right|_{m_{\imath}} & =0
\end{aligned}
$$

The running of $\Delta Z$ 's is extracted from the self-energy diagrams of the neutral vector bosons with an internal b-quark in the loop. There are of course other diagrams involving operators with explicit suppression factors of $1 / m_{t}$, which we neglect. A simple computation, using dimensional regularization, gives

$$
\begin{aligned}
\mu \frac{d}{d \mu} \Delta Z_{W^{ \pm}} & =0 \\
\mu \frac{d}{d \mu} \Delta Z_{W^{3}} & =-\left(\frac{N_{c}}{3}\right) \frac{g^{2}}{16 \pi^{2}} \\
\mu \frac{d}{d \mu} \Delta Z_{W^{3} B} & =\left(\frac{N_{c}}{3}\right) \frac{2 Y_{L} g g^{\prime}}{16 \pi^{2}} \\
\mu \frac{d}{d \mu} \Delta Z_{B} & =-\left(\frac{N_{c}}{3}\right) \frac{4\left(Y_{L}^{2}+Y_{R}^{2}\right) g^{\prime 2}}{16 \pi^{2}},
\end{aligned}
$$

where $Y_{L}=1 / 6$ and $Y_{R}=-1 / 3$ stand for the hypercharge of the left and right handed $b$-quarks, respectively, and $N_{c}=3$ is the number of colors (for a heavy lepton one has the same but with $Y_{L}=-1 / 2, Y_{R}=-1$ and $\left.N_{c}=1\right)$. Solving, we have

$$
\begin{aligned}
\left.\Delta Z_{W^{ \pm}}\right|_{\mu} & =0 \\
\left.\Delta Z_{W^{3}}\right|_{\mu} & =\left(\frac{N_{c}}{3}\right) \frac{g^{2}}{16 \pi^{2}} \log \frac{m_{t}}{\mu} \\
\left.\Delta Z_{W^{3} B}\right|_{\mu} & =-\left(\frac{N_{c}}{3}\right) \frac{2 Y_{L} g g^{\prime}}{16 \pi^{2}} \log \frac{m_{t}}{\mu} \\
\left.\Delta Z_{B}\right|_{\mu} & =\left(\frac{N_{c}}{3}\right) \frac{4\left(Y_{L}^{2}+Y_{R}^{2}\right) g^{\prime 2}}{16 \pi^{2}} \log \frac{m_{t}}{\mu}
\end{aligned}
$$

As advertised above, the $\Delta Z$ 's do not get additional QCD corrections, in the leading-log approximation. 
The last step is the calculation of the vector bosons' renormalized two-point functions in the effective theory. Although we originally intended to compute this at $\mu$ of the order of the weak vector boson's mass, we will keep $\mu$ arbitrary. The explicit cancellation of $\mu$ dependence is a check on the calculation. It is the lack of QCD effects to this order that allows us to explicitly keep track of the renormalization point. Since the $b$-quark mass is neglected relative to the $W$-boson mass, the two point functions are proportional to $p^{2}$, where $p_{\mu}$ is the momentum of the vector boson. To extract the physical mass we need this at $p^{2}=M_{\text {phys }}^{2}$. Thus, effectively, these contributions act like additional wavefunction renormalizations, which we label by a supercript $(b)$ :

$$
\begin{aligned}
\left.\Delta Z_{W^{ \pm}}^{(b)}\right|_{\mu} & =0 \\
\left.\Delta Z_{W^{3}}^{(b)}\right|_{\mu} & =-\left(\frac{N_{c}}{3}\right) \frac{g^{2}}{16 \pi^{2}}\left(\log \frac{M_{W}}{\mu}-\frac{5}{6}\right) \\
\left.\Delta Z_{W^{3} B}^{(b)}\right|_{\mu} & =\left(\frac{N_{c}}{3}\right) \frac{2 Y_{L} g g^{\prime}}{16 \pi^{2}}\left(\log \frac{M_{W}}{\mu}-\frac{5}{6}\right) \\
\left.\Delta Z_{B}^{(b)}\right|_{\mu} & =-\left(\frac{N_{c}}{3}\right) \frac{4\left(Y_{L}^{2}+Y_{R}^{2}\right) g^{\prime 2}}{16 \pi^{2}}\left(\log \frac{M_{W}}{\mu}-\frac{5}{6}\right)
\end{aligned}
$$

The calculation of physical masses, expressed in terms of $G_{\mu}, \alpha$ and $M_{Z}$, can now be done directly from the effective lagrangian. After some trivial algebra we obtain

$$
\begin{aligned}
M_{W+, \text { phys }}^{2}= & \cos ^{2} \theta_{0} M_{Z, \text { phys }}^{2}\left[1-\left.\left(\frac{\cos ^{2} \theta_{0}}{\cos 2 \theta_{0}}\right) \Delta \rho\right|_{\mu=M_{W}}\right. \\
& \left.+\left[\left(\Delta Z_{W^{3}}+\Delta Z_{W^{3}}^{(b)}\right)-\left(\Delta Z_{W^{ \pm}}+\Delta Z_{W^{ \pm}}^{(b)}\right)\right]-\left(\frac{\sin 2 \theta_{0}}{\cos 2 \theta_{0}}\right) \Delta Z_{W^{3} B}\right] \\
= & \cos ^{2} \theta_{0} M_{Z, \text { phys }}^{2}\left[1-\left.\left(\frac{\cos ^{2} \theta_{0}}{\cos 2 \theta_{0}}\right) \Delta \rho\right|_{\mu=M_{W}}\right. \\
& \left.+\frac{\alpha}{8 \pi \sin ^{2} \theta_{0}}\left(\ln m_{t}^{2} / M_{Z}^{2}+5 / 3\right)+\frac{\alpha}{12 \pi \cos 2 \theta_{0}}\left(\ln m_{t}^{2} / M_{Z}^{2}+5 / 3\right)\right]
\end{aligned}
$$

where $\sin ^{2} 2 \theta_{0}=4 \pi \alpha / \sqrt{2} G_{\mu} M_{Z, \text { phys }}^{2}$, and by $\left.\Delta \rho\right|_{\mu=M_{W}}$ we mean $\Delta \rho$ as given in eq. (3.7), with $k=3 \alpha / 16 \pi \sin ^{2} \theta_{0}$, but with the replacement $\bar{\alpha}_{s}\left(m_{l}\right) \rightarrow \bar{\alpha}_{s}\left(M_{W}\right)$. In comparing this result with others in the literature one must bare in mind that only the leading-log contribution of the third generation quarks has been included here.

\section{Conclusions and Sub-Leading-Logs}

We have demonstrated the use of effective field theories for the resummation of possibly large logs from QCD in corrections to electroweak parameters. We showed how the 
calculation can be carried out to arbitrary accuracy by incorporating sub-leading power corrections, i.e., corrections suppressed by the heavy mass.

We have not considered sub-leading log improvements on these calculations. The results in eqs. (3.7) and (3.16) include a term, proportional to $m_{t}^{2}$, which is leading-log even if it does not contain any explicit logs. For large $m_{t}$ this term is certainly the dominant contribution to $\Delta \rho$ and $M_{W, \text { phys }}^{2}$. Terms of order $m_{t}^{2}$ can only appear from matching at the $t$-quark scale - it is impossible to generate positive powers of the large mass once in the effective theory (otherwise, the whole notion of effective theory would be useless!).

The next-to-leading-log correction -of order $\alpha_{t}-$ to the term of order $m_{t}^{2}$ in eqs. (3.7) and (3.16) is numerically as important as the order $m_{b}^{2}$ terms which occupied us in the bulk of this paper. There is an important lesson about it that we have learnt in this work: the whole correction of order $\alpha_{s} m_{t}^{2}$ comes from matching. Once the two-loop matching condition is calculated, the strong coupling constant in the coefficients of operators in the effective theory should be taken to be the running coupling constant evaluated at the top scale, $\bar{\alpha}_{s}\left(m_{t}\right)$. Concretely, the corrections to $\rho$ of order $m_{t}^{2}$ up to order $\alpha_{t}$ can be written

$$
\Delta \rho=\frac{3 \alpha}{16 \pi M_{W}^{2} \sin ^{2} \theta_{W}} m_{t}^{2}\left[1+\frac{\alpha_{s}}{\pi} \kappa\right] .
$$

The two-loop diagram giving rise to the order $\alpha_{s}$ correction has been calculated[15]

$$
\kappa=-\frac{2 \pi^{2}+6}{9}
$$

There has been some controversy ${ }^{2}$ over how to choose the scale at which $\alpha_{\mathrm{s}}$ is evaluated in eq. (5.2). This is not a trivial question, as the loop integrals that lead to eqs. (5.1) and (5.2) contain internal $b$ - and $t$-quarks, and the momentum transfer $q^{2}$ can be either negligible, as in the case of $\Delta \rho$, or of the order of $M_{Z}^{2}$, as when computing the physical masses. Also, and possibly more problematic, is the issue of gauge and scheme dependence, of which the authors of ref. [15] have little to say. As we have seen, in the language of effective lagrangians the answer is immediate. We have seen that one should replace $\alpha_{s}$ in eq. (5.2) by $\bar{\alpha}_{s}\left(m_{t}\right)$, where $\bar{\alpha}_{t}$ is the running coupling constant of the effective theory below $m_{t}$.

\footnotetext{
${ }^{2}$ See, for example, the review in ref. [11].
} 


\section{Acknowledgements}

We thank A. Falk, M. Dugan, H. Georgi and L. Randall for discussions. B.G. would like to thank the Alfred P. Sloan Foundation for partial support. This research is supported in part by the National Science Foundation under grant PHY-87-14654, by the Texas National Research Laboratory Commission, under grant RGFY9106 and by the Department of Energy under contract DE-AC35-89ER40486. 


\section{References}

[1] Z Physics at LEP1, G.Altarelli, R.Kleiss, and C.Verzegnassi, eds., CERN report, CERN-89-08, 1989

[2] D.C.Kennedy and B.W.Lynn, Nucl. Phys. B322 (1989)1

[3] A. Sirlin, Nucl. Phys. B71 (1974) 29; Nucl. Phys. B100 (1975) 291; Phys. Rev. D22 (1980) 971

M. Veltman, Acta Phys. Pol. B8 (1977) 475; Nucl. Phys. B123 (1977) 89;

W. Marciano and A. Sirlin, Phys. Rev. D22 (1980) 2695; erratum-ibid. D31 (1980) 213;

S.Dawson, J.S.Hagelin and L.Hall, Phys. Rev. D23 (1981) 2666

[4] M.Golden and L.Randall, Nucl. Phys. B361 (1991) 3.

[5] M.E.Peskin and T.Takeuchi, Phys. Rev. Lett. 65 (1990) 964;

B. Holdom and J. Terning, Phys. Lett. 247B (1990) 88.;

G. Altarelli, R. Casalbuoni, F. Feruglio and G. Gatto, Phys. Lett. 245B (1990) 669;

G. Altarelli and R. Barbieri, Phys. Lett. B253 (1991) 161.

[6] B. W. Lynn, M. E. Peskin and R. G. Stuart, Trieste Electroweak 213 (1985), also SLAC preprint SLAC-PUB-3725(1985)

[7] S. Weinberg, Phys. Rev. D19 (1979) 1277;

L. Susskind, Phys. Rev. D20 (1979) 2619;

P. Sikivie, L. Susskind, M. Voloshin and V. Sakharov, Nucl. Phys B173 (1980) 189

[8] M.B.Einhorn, D.R.T.Jones and M.Veltman, Nucl. Phys. B191 (1981)146

[9] H.Georgi, Weak Interactions and Modern Particle Theory (Benjamin/Cummings, Menlo Park, CA, 1984)

[10] A.Cohen, H.Georgi and B.Grinstein, Nucl. Phys. B232 (1984) 61

[11] M. Consoli and W. Hollik, Electroweak Radiative Corrections for Z Physics, in CERN report CERN 89-08 Vol. 1

[12] A. Falk and B. Grinstein, Phys. Lett. 247B (1990) 406

[13] B. Grinstein and L. Randall, Phys. Lett. 217B (1989) 335

[14] F. Jegerlehner, Z. Phys. C 32 (1986) 195

[15] A. Djouadi and C. Verzegnassi, Phys. Lett. 195B (1987) 265;

A. Djouadi, Nuovo Cim. 100A (1988) 357 


\section{Figure Captions}

Fig. 1. Feynman diagrams representing the fermion interactions of the lagrangian in the full theory. Here and throughout, a wavy line represents an electroweak gauge boson and a solid directed line is a fermion.

Fig. 2. Feynman diagrams representing the light fermion (" $b$ ") interactions of the effective theory, once the heavy fermion has been integrated out. The solid dot represents the new higher dimension operaors.

Fig. 3. The one loop contribution to $\left.\Delta M_{W^{ \pm}}^{2}\right|_{\mu=m_{h}}$.

Fig. 4. One loop contributions to $\left.\Delta M_{W^{3}}^{2}\right|_{\mu=m_{h}}$.

Fig. 5. Feynman diagram giving the one-loop mixing of the operators in eqs. (2.6) and $(2.10 a)$.

Fig. 6. Feynman diagram giving the one loop running of the operator in (2.10b)

Fig. 7. One-loop contribution to $\rho$ of the six-fermion operators defined in eq. (2.13).

Fig. 8. One-loop diagrams contributing to the renormalization of the operators in eq. (2.6). A spring line represents a gluon. The heavy dot stands for the composite operators.

Fig. 9. One-loop diagrams that give rise to the running of the four-quark operators in the lagrangian (3.8). 


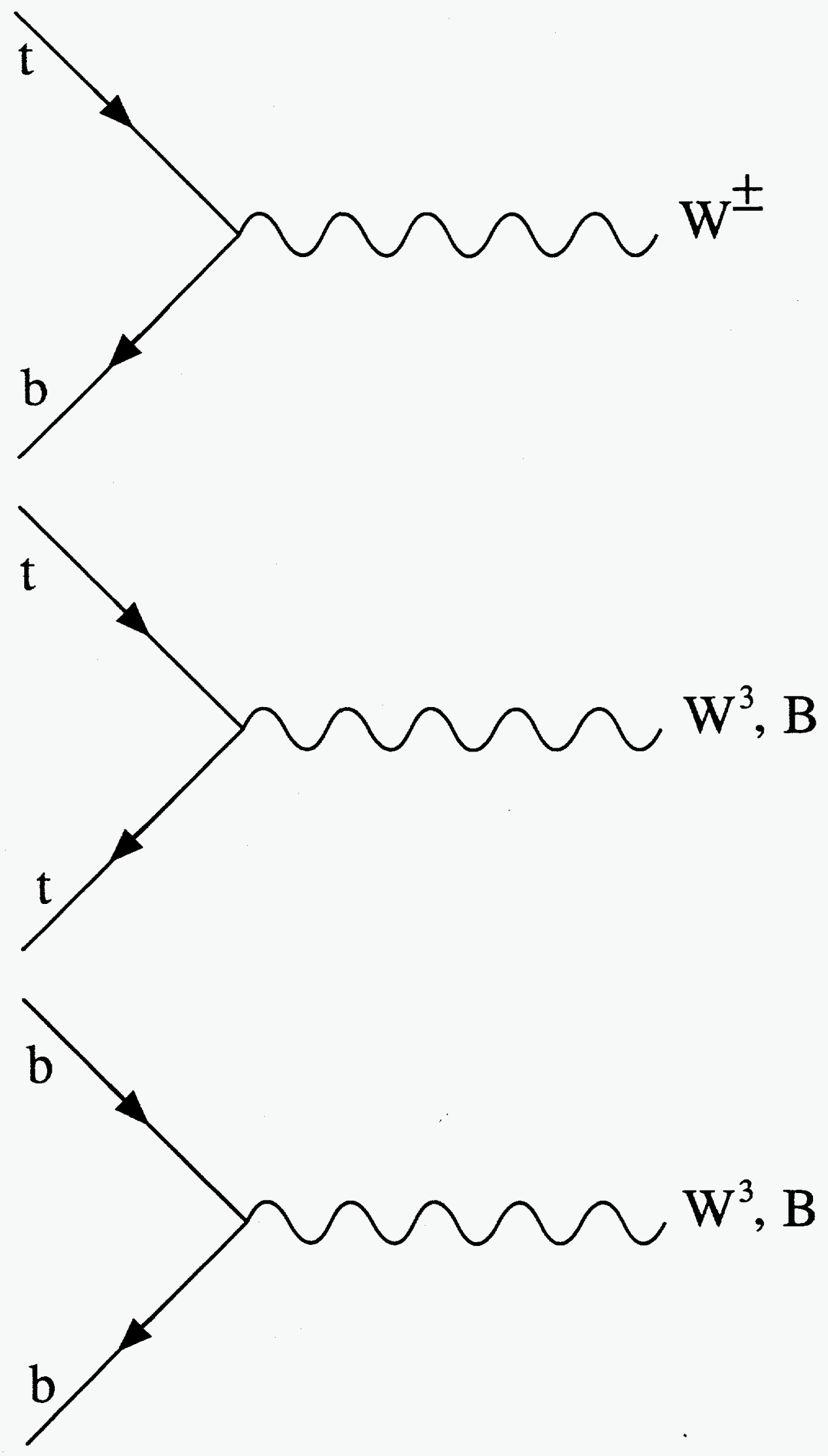

GRINSTEN W WANG: Floure 1 

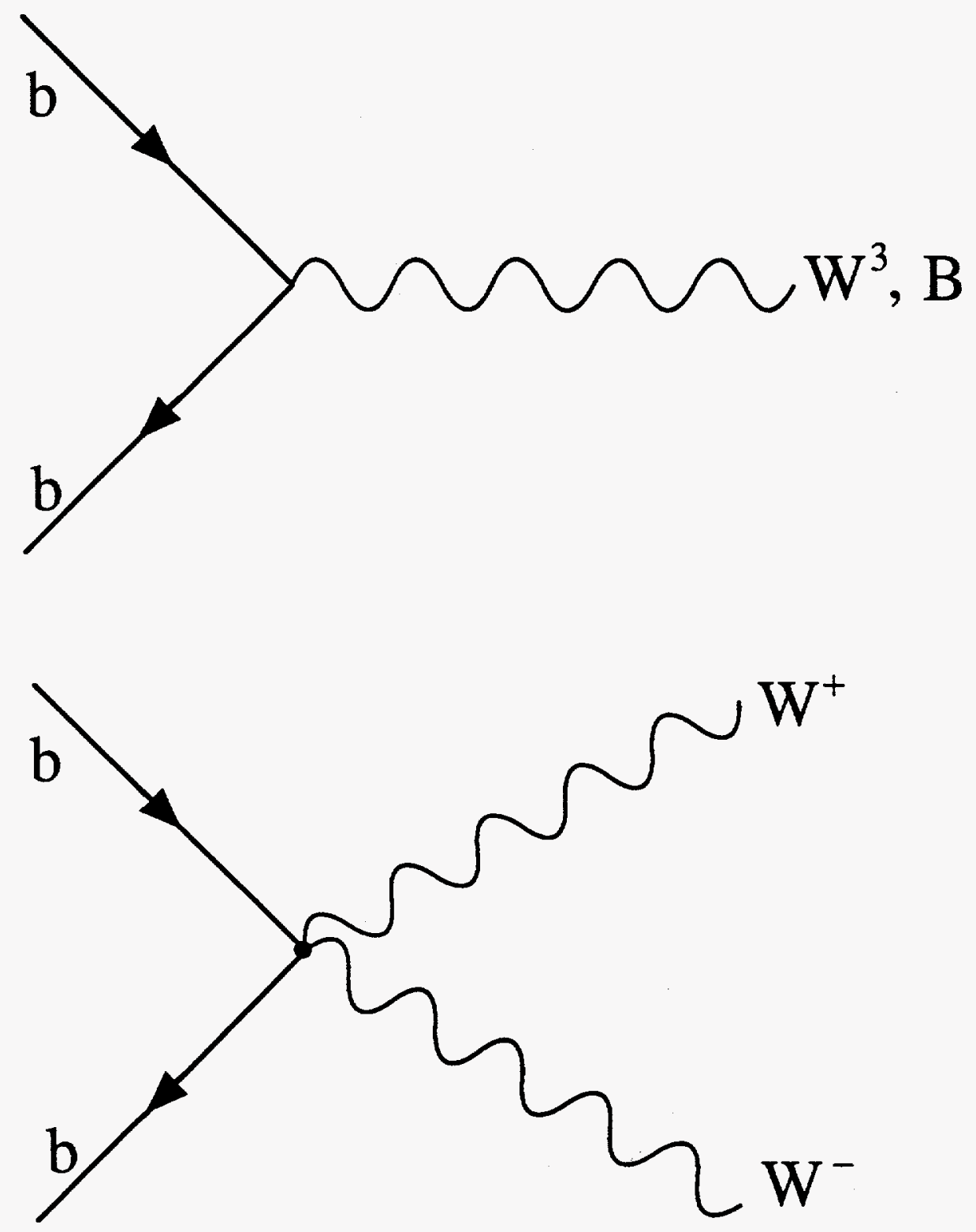


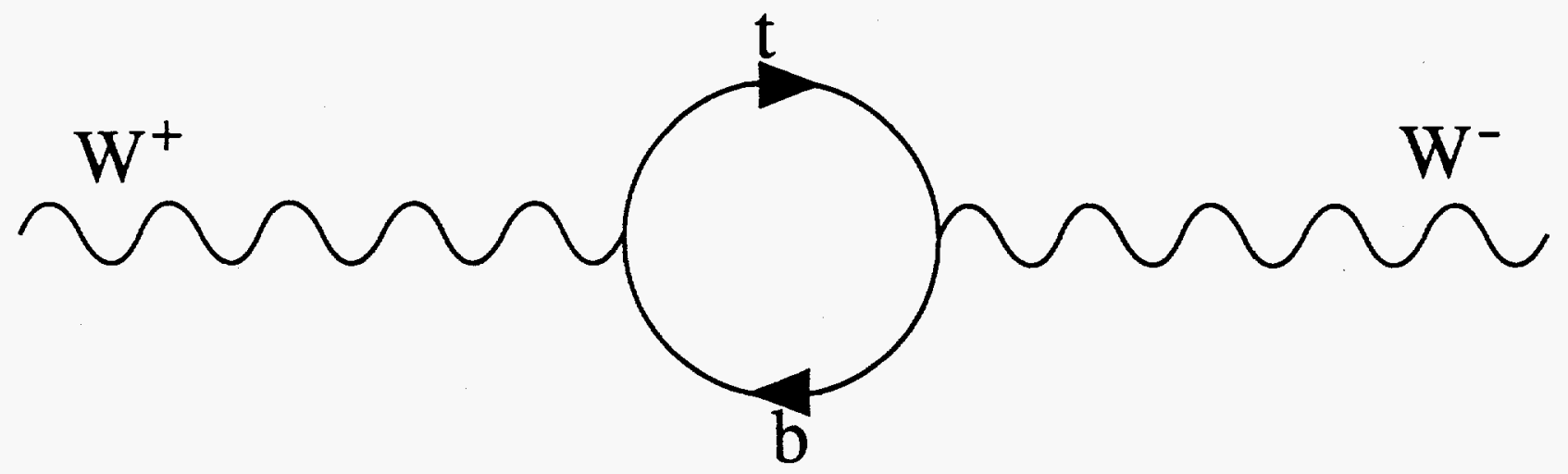

GRINSTEIN \& WANG: FOUNO 3 

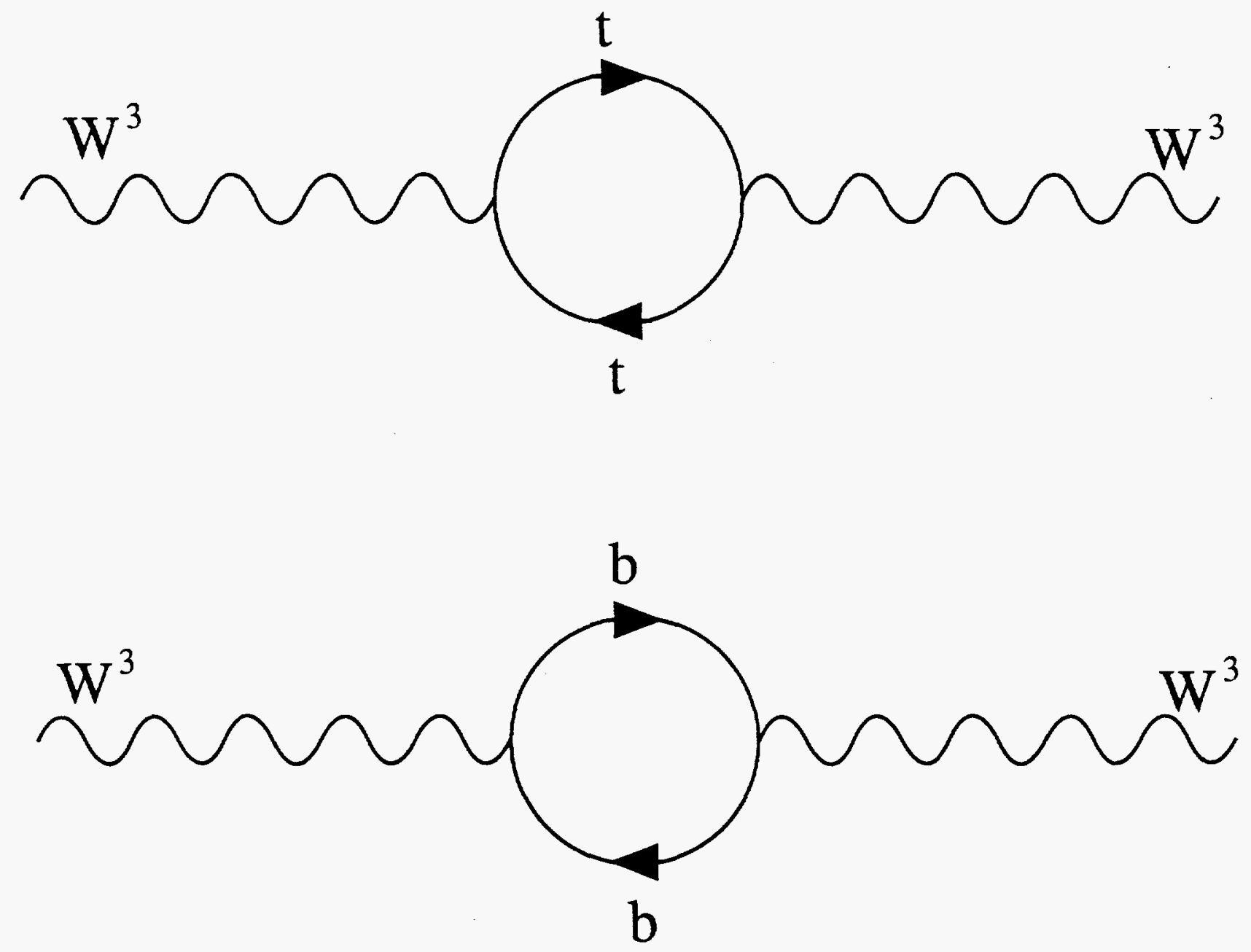


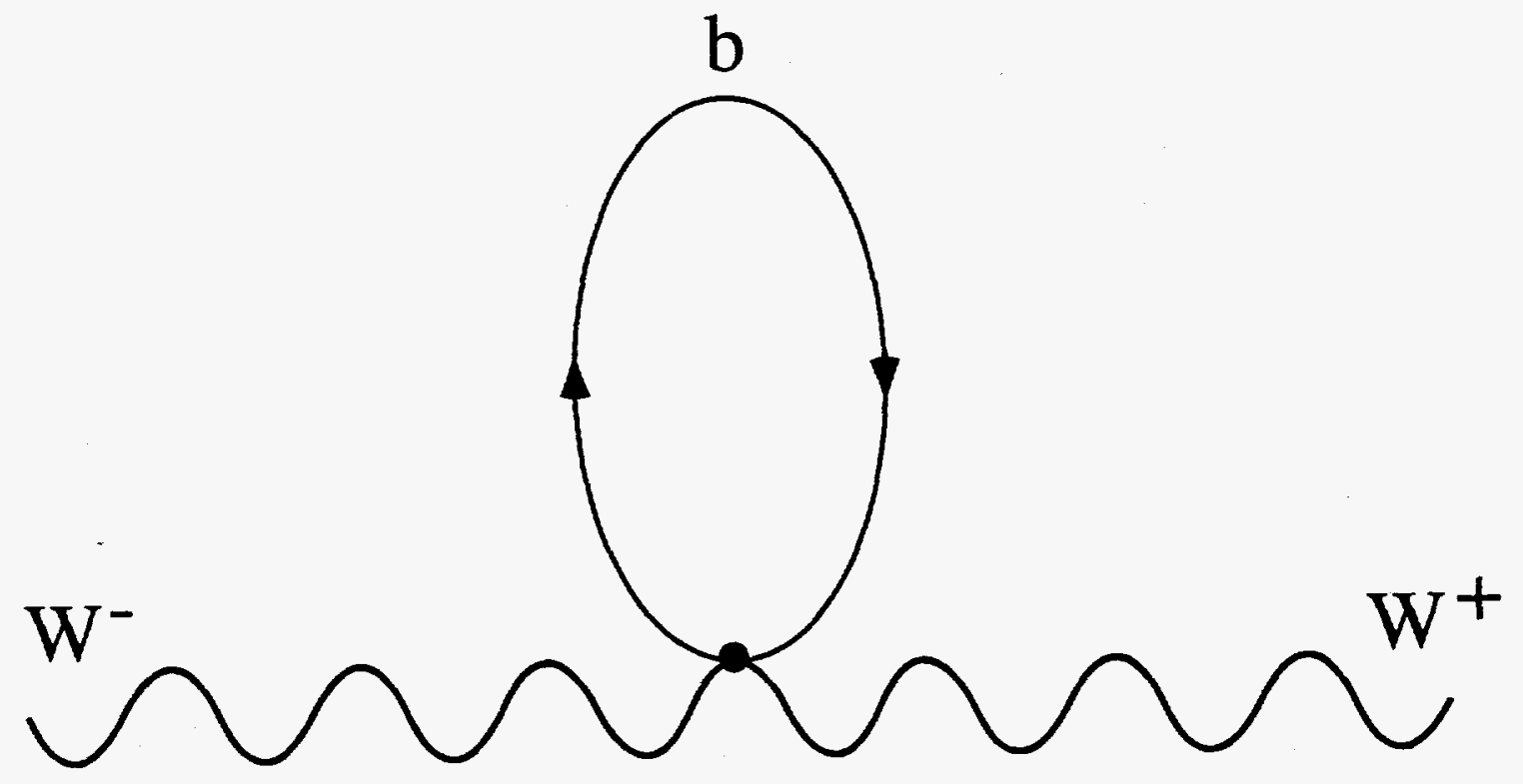




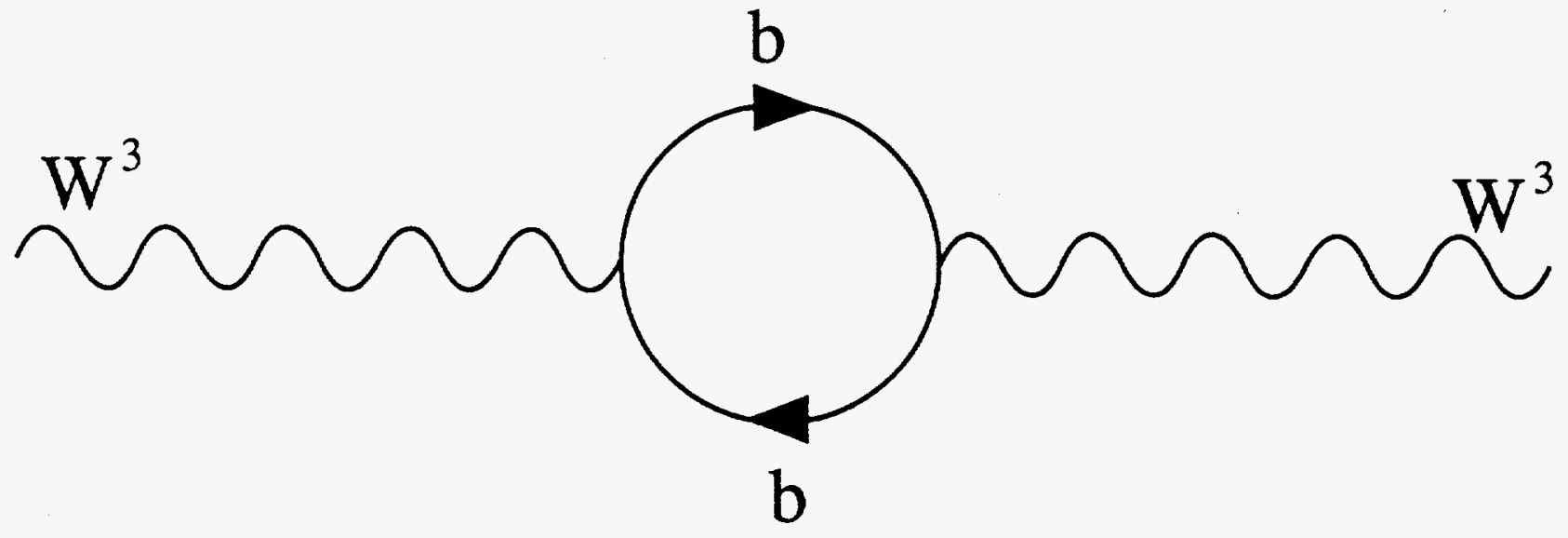




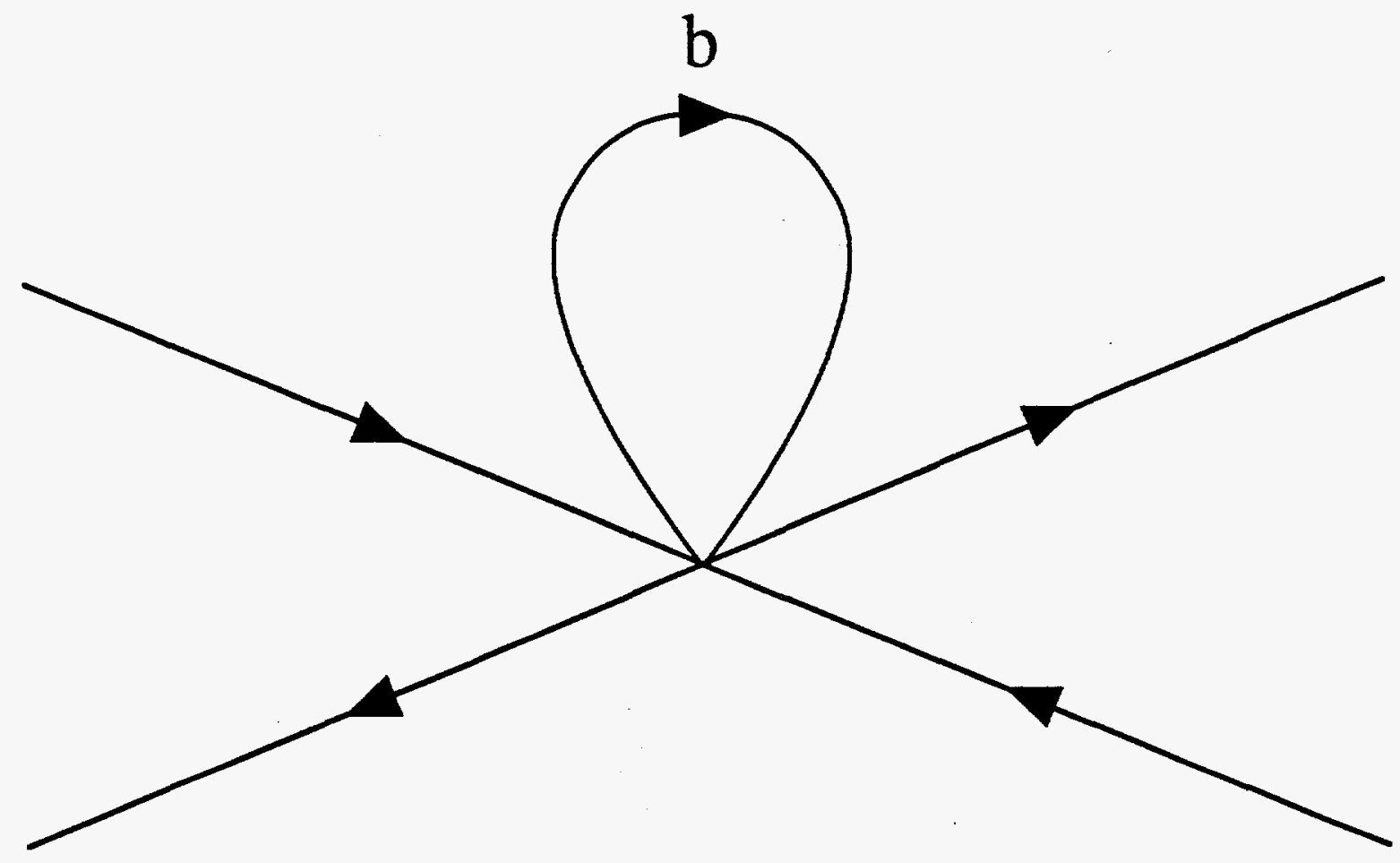

GRINSTEN \& WANG: FGUTO 7 

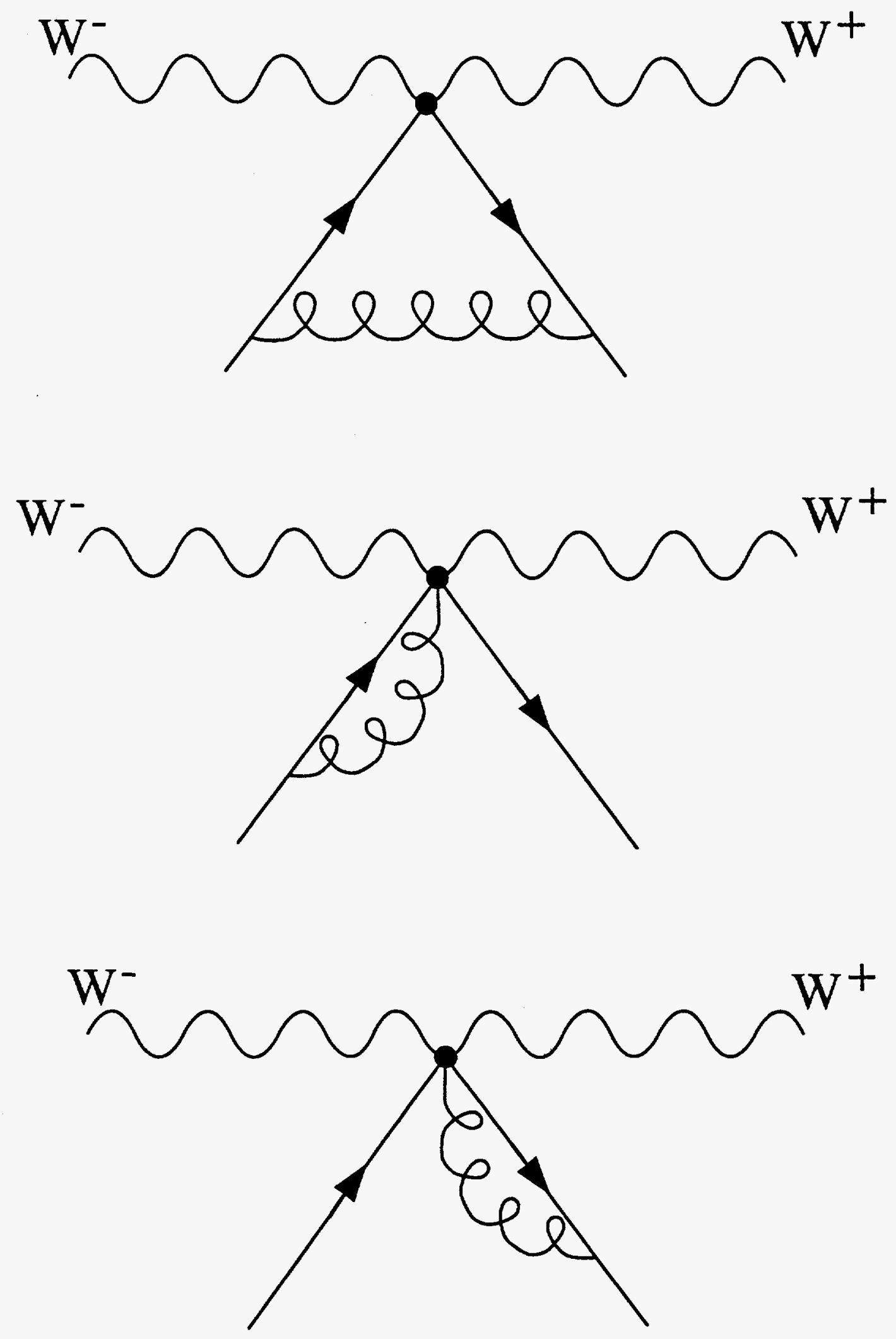

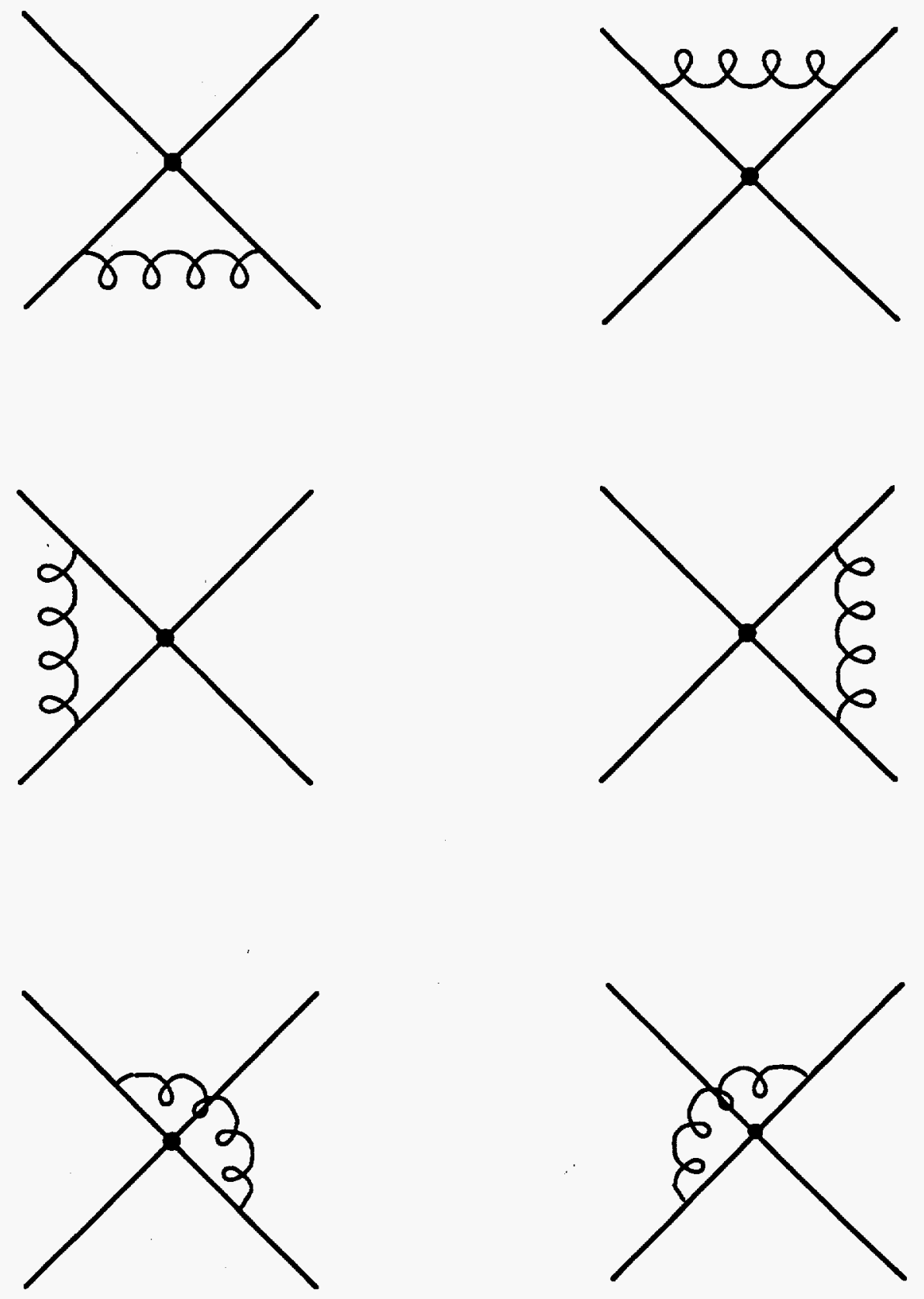